\section{ADHS macht weniger empfindlich gegen Schmerz}

\author{
Viele Eltern von Kindern mit ADHS berichten von einer reduzierten Schmerz- \\ wahrnehmung. In einer aktuellen Studie aus Deutschland wurde deshalb die \\ Schmerzempfindlichkeit von ADHS-Patienten und ihre Beeinflussbarkeit \\ durch eine Therapie mit Methylphenidat (MPH) untersucht.
}

E ür die Studie wurden 130 Kinder im Alter von 7-17 Jahren mit ADHS ausgewählt, deren Eltern über eine Schmerzunterempfindlichkeit ihrer Sprösslinge berichteten. Alle Teilnehmer stammten aus der „Studie zur Gesundheit von Kindern und Jugendlichen in Deutschland “ (KiGGS), die von 2003-2006 bei 1.7641 Kindern und Jugendlichen im Alter von 0-17 Jahren erhoben worden war. Die Hälfte der Probanden mit ADHS wurde mit MPH therapiert, die andere Hälfe nicht. Sie wurden verglichen mit einer Kontrollgruppe aus der KiGGS-Studie bestehend aus 130 Kindern ohne ADHS.

Die Wissenschaftler um Nicole Wolff befragten nun die Eltern, ob ihre Kinder sich in den letzten 3 Monaten über Schmerzen beklagt hatten, und wenn ja, wo die Schmerzen lokalisiert waren. Sie wurden gebeten, die Schmerzintensität auf einer Schmerzskala von 0 (schmerzunterempfindlich) bis 100 (extrem schmerzempfindlich) anzugeben. Kinder und Jugendliche im Alter von 11-17 Jahren beurteilten ihre Schmerzempfindlichkeit selbst.

Die Autoren stellten fest, dass es keine Unterschiede hinsichtlich der Schmerzempfindlichkeit bezüglich des $\mathrm{Ge}$ schlechts und des Alters gab. Die Lokalisation des Schmerzes unterschied sich nicht in den drei Gruppen (ADHS mit Medikation, ADHS ohne Medikation, Kontrollgruppe). Hinsichtlich der Wahrnehmung der Schmerzintensität hingegen zeigten sich signifikante Unterschiede zwischen den drei Gruppen: Kinder mit ADHS ohne Medikation zeigten die geringste Schmerzwahrnehmung, Kinder mit ADHS unter MPH zeigten eine deutlich höhere Schmerzwahrnehmung, die mit der von Kindern ohne ADHS vergleichbar war.

Die Autoren schließen aus ihren Ergebnissen, dass die Schmerzunterempfindlichkeit durch eine MPH-Therapie erfolgreich therapiert wird und auf ein
„Normalniveau“ ansteigt. Sie vermuten, dass bei Kindern mit ADHS ein Defizit an zerebralen Opioiden vorliegt, das mit Hilfe von MPH korrigiert wird. Sie sehen außerdem einen Zusammenhang zwischen der erhöhten Unfallgefährdung der Kinder mit ADHS und ihrer Schmerzunterempfindlichkeit.

Wolff $\mathrm{N}$ et al. Reduced pain perception in children and adolescents with ADHD is normalized by methylphenidate. Child Adolesc Psychiatry Ment Health 2016;10:24

\section{Kommentar}

Schmerzunterempfindlichkeit war bisher nie zentrales Thema in Studien über die ADHS-Symptomatik. Jedoch berichteten viele Eltern von Kindern mit ADHS von einer reduzierten Schmerzwahrnehmung. „Hart im Nehmen" sei das Kind und darüber hinaus noch ausgesprochen kälteunempfindlich, was vor allem im Winter zu heftigen Auseinandersetzungen bei der Kleiderauswahl führe. Meist handelt es sich hier um Jungen, meistens um Kinder mit einer ADHS vom kombinierten Typ. Seltener wird von diesem Phänomen bei der verträumten, rein unaufmerksamen Form berichtet.

In der vorliegenden Studie wird dieser klinische Eindruck bestätigt. Die Ursache für die Schmerzunterempfindlichkeit bleibt unklar. Es gibt keine objektiven Parameter wie eine Reduzierung der sensorisch evozierten Potenziale, eine Verlangsamung der sensiblen Nervenleitgeschwindigkeit oder Auffälligkeiten in den sensiblen Arealen im funktionellen MRT. Es wurde auch von einer möglichen positiven Korrelation mit Impulsivität und Hyperaktivität berichtet.

Der Zusammenhang einer Schmerzunterempfindlichkeit mit erhöhter Unfallneigung ist möglich. Es sind aber noch andere Ursachen denkbar, die zu einer erhöhten Unfallneigung führen könnten, wie Unaufmerksamkeit oder erhöhte Risikobereitschaft bedingt durch eine Dysfunktion im Belohnungszentrum.

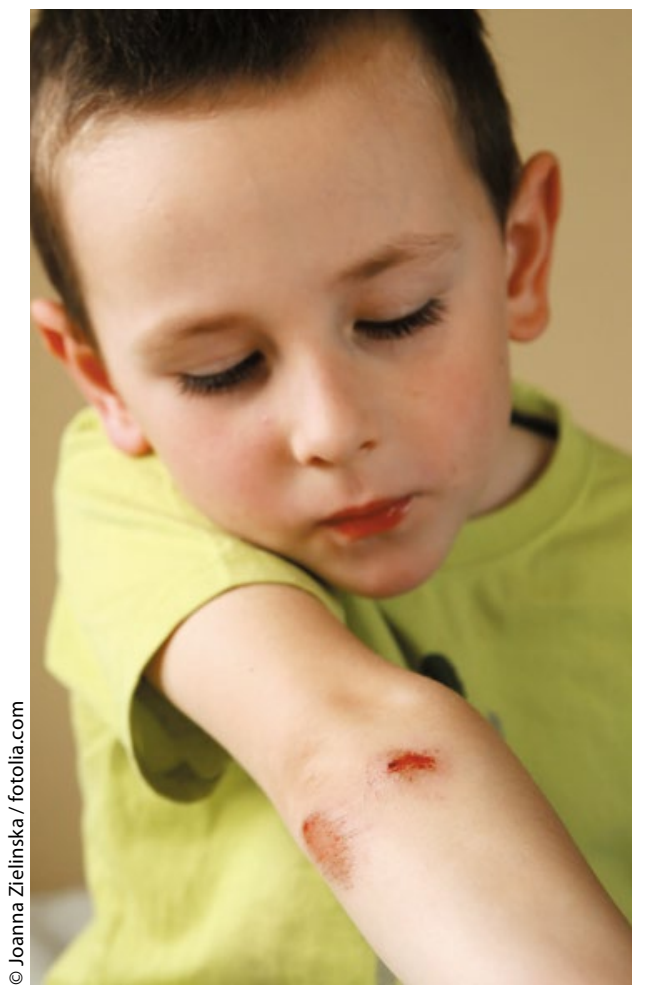

Für Jungen mit ADHS ist solch eine Verletzung häufig nicht sehr schmerzhaft.

Interessant ist der durch die Studienergebnisse belegt positive Einfluss auf die Schmerzwahrnehmung durch die Medikation mit Methylphenidat. Auch hier bleibt aber unklar, wie die Wirkung zustande kommt. Die Autoren vermuten einen Einfluss des MPH auf das opioide System. Denkbar wäre aber auch eine Verbesserung der Schmerzwahrnehmung durch Reduzierung der Kernsymptomatik der ADHS, vor allem der Reduzierung der erhöhten Ablenkbarkeit.

Auch wenn die Schmerzunterempfindlichkeit nicht spezifisch für das Vorliegen einer ADHS ist, so sollte sie doch dem Symptomenkatalog hinzugefügt werden. Weiterer Forschung bleibt es vorbehalten, zu untersuchen, ob es sich hier um eine Subgruppe der ADHS handelt, ob sich die kombinierte Form hinsichtlich des Schmerzempfindens von der rein unaufmerksamen Form unterscheidet und außerdem, ob eine Korrelation von Schmerzunterempfindlichkeit im Kindesalter mit erhöhter Unfallgefährdung und eventuell auch Suchtproblematik im Jugendlichen- beziehungsweise Erwachsenenalter besteht.

Dr. Kirsten Stollhoff 